\title{
Modelling and analysis of DCIS detection and reduction in invasive carcinoma - a review
}

\author{
S. W. Duffy \\ Department of Cancer Screening, Cancer Research UK for Epidemiology, Mathematics and Statistics, \\ Wolfson Institute of Preventive Medicine, London, UK.
}

\begin{abstract}
Although much has been written about overdiagnosis in mammographical screening, analytical estimates of the extent of overdiagnosis are rare in the literature. Estimates specific to ductal carcinoma in situ (DCIS) and the implications for future invasive disease are even more difficult to find. In this paper, we review studies of incidence of DCIS within breast screening programmes and its association with subsequent incidence of invasive breast cancer. Although sparse, published results suggest that the majority of DCIS cases have the propensity to progress to invasive disease.
\end{abstract}

Keywords: Breast cancer screening; DCIS; Invasive carcinoma

\section{Introduction}

Concerns have been expressed about the possibility of overdiagnosis in breast cancer screening [1]. There is particular interest in the potential for overdiagnosis of ductal carcinoma in situ (DCIS) [2]. This interest arises from the following observations:

1. Incidence of DCIS has risen dramatically in proportional terms since the onset of mammographical screening programmes [3].

2. Retrospective studies of DCIS cases which were previously misdiagnosed as benign disease and untreated found that only a minority progressed to invasive disease over a long period of follow-ups [4].

3. Autopsy studies of women with no diagnosis of breast cancer during their lifetime suggest a prevalence of occult DCIS of between $9 \%$ and $15 \%$ [5].

Correspondence to: S. W. Duffy, Department of Cancer Screening, Cancer Research UK for Epidemiology, Mathematics and Statistics, Wolfson Institute of Preventive Medicine, Charterhouse Square, London EC1M 6BQ, UK. E-mail: stephen.duffy@cancer.org.uk; Tel: +1 207014 0252; Fax: +1 20 70140258

Publication date 29/07/05

BCO/349/2005/FO
Here, we review the published evidence on DCIS and its potential to progress to invasive disease if left untreated. Since it is not considered ethical to leave DCIS untreated, the evidence is necessarily indirect, involving deductions from rates of DCIS diagnosed, or inference from outcomes in treated DCIS. We divide the studies reviewed into two groups: clinicopathological studies relating features of tumour and treatment to prognosis and progression in treated DCIS; and inferential estimation of rates of progression from data on numbers of invasive and in situ tumours diagnosed in screening programmes.

\section{Clinicopathological studies}

A large number of studies have been conducted on the clinical and pathological features of DCIS which relate to future clinical outcomes. For brevity, we shall confine our review to the major implications of the most recent studies. What is very clear in the first instance is that a substantial minority of DCIS cases recur or progress despite treatment [6]. In a randomized trial of local excision against local excision plus radiotherapy, $19 \%$ of those treated with local excision alone had a local recurrence within a median 
follow-up of 5.4 years [6]. Rates were considerably higher in younger patients, larger tumours, highgrade tumours and cases where the surgical margin showed tumour involvement. These prognostic factors in DCIS have been long-established [7], but the high absolute rates of recurrence after treatment strongly suggest that in the absence of treatment, progression would be considerably more common.

This is supported by the findings of Evans et al. [8], who observed that the majority of screen-detected DCIS cases were of high grade and necrotic. This suggests that a high proportion of DCIS cases detected at screening are at risk of progression to invasive cancer. Similar observations have been made in other studies of tumour biology, as reviewed by Feig [9].

Clinicopathological studies therefore indicate that the presence of DCIS confers substantial risk of invasive breast cancer. Until the discovery of markers which reliably distinguish progressive and nonprogressive lesions, the clinical issue of importance is not whether to treat, but how best to decide individual treatment to prevent subsequent invasive disease [10]. In the meantime, estimation from multistate modelling of data on rates of invasive cancer and DCIS from screening programmes may at least help to quantify the proportion of DCIS tumours which are non-progressive, and so give an estimation of the potential size of the overdiagnosis/ overtreatment problem.

\section{Estimation of rates from tumour progression models}

The most direct example of this approach is that of Yen et al. [2] who used data from screening programmes in Europe, the USA and Australia to estimate the parameters of a mover-stayer model as in Fig. 1. In this model, a woman may remain free of breast cancer all her life, may develop non-progressive DCIS which either remains in the breast or spontaneously regresses, or may develop progressive DCIS which in turn may progress to invasive disease.

For any given case of DCIS detected at screening, treated, and with no observed recurrence or progression so far, we cannot know what would have happened if that case had not been treated. This means that we cannot identify individual nonprogressive cases. However, we can estimate the proportion of such cases from the rates of DCIS and invasive cancer observed at prevalence (first) and incidence (later) screens.

Table 1 shows the rates per thousand of DCIS at prevalence and incidence screens used by Yen and colleagues for estimation [2]. Table 2 shows the estimates derived from these data. The major implications are that at a prevalence screen, $37 \%$ of
DCIS cases are estimated to be non-progressive (around $5 \%$ of all tumours diagnosed), and at incidence screen $4 \%$ of DCIS cases are non-progressive (less than $1 \%$ of all tumours).

Less directly, but more simply, Paci et al. [11] estimated overdiagnosis rates in the Florence breast screening programme, including and excluding DCIS. They compared incidence of breast cancer in Florence in 1990-1999 after the introduction of screening in 1990, with incidence observed before the introduction, in 1985-1989. Clearly, some excess incidence would be expected in the screening period even if there were no overdiagnosis at all, since some tumours which would otherwise have arisen clinically after 1999 would be diagnosed early by screening during 1990-1999. Paci and colleagues estimated this number using estimates of the average lead time.

Their results are shown in Table 3. An observed significant excess of $11 \%$ in invasive tumours only became a non-significant $2 \%$ excess after removal of the 'lead time' tumours. When in situ cases were included, there remained a significant $5 \%$ excess incidence after removal of the tumours anticipated due to lead time. These results suggest that overdiagnosis is largely confined to DCIS and is not of a magnitude which would contraindicate screening.

Finally, an interesting approach was employed by McCann et al. [12], who extrapolated pre-screening trends in the UK to the screening epoch, the 1990s. Comparing actual incidence in the screening epoch

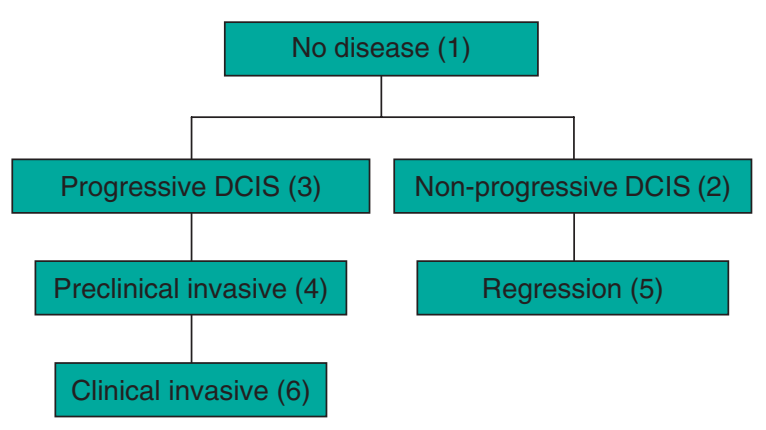

Figure 1.

Representation of a mover-stayer model for DCIS. A woman may remain disease-free, may develop non progressive DCIS or may develop progressive DCIS. If she develops progressive DCIS, this may progress to asymptomatic and subsequently symptomatic invasive breast cancer, although this progression may be arrested if the disease is detected by screening and treated while still DCIS. If she develops non-progressive DCIS, the disease cannot progress to invasive cancer and may regress. If it is detected by screening before it regresses, this consitutes overdiagnosis. The rates of development of nonprogressive and progressive DCIS, and rates of further progression of the latter were estimated from the data sources in Table 1. 
with that predicted from the pre-screening trends, they found an excess of all tumours in the screening age group, 50-64, peaking in the early 1990s and a deficit in incidence of invasive tumours at ages $65-69$, peaking in the late 1990s. Application of a range of plausible lead times to the early excess of invasive cancers alone, in the cohorts invited to screening, could not fully account for when the DCIS cases in the early excess period were also included.

Table 1. Detection rates of DCIS and invasive breast cancer at prevalence and incidence screens in the data used to estimate the parameters of the mover-stayer model of Yen and colleagues [2].

\begin{tabular}{llll}
\hline & Tumour type & $\begin{array}{l}\text { Prevalence } \\
\text { screen } \\
\text { rate/1000 }\end{array}$ & $\begin{array}{l}\text { Incidence } \\
\text { screen } \\
\text { rate/1000 }\end{array}$ \\
\hline Data source & DCIS & 0.4 & 0.4 \\
$\begin{array}{l}\text { Swedish } \\
\text { ages 40-49 }\end{array}$ & Invasive & 1.7 & 2.4 \\
Swedish & DCIS & 0.7 & 0.6 \\
Two-County, & Invasive & 4.0 & 2.6 \\
ages 50-59 & DCIS & 0.8 & \\
Swedish & Invasive & 8.1 & 0.5 \\
Two-County, & DCIS & 1.1 & \\
ages 60-69 & & 0.8 \\
UK programmes & Invasive & 4.9 & 4.0 \\
The Netherlands & DCIS & 0.8 & 0.5 \\
programmes & Invasive & 5.1 & 2.8 \\
South Australia & DCIS & 1.2 & 0.6 \\
& Invasive & 5.8 & 2.8 \\
New York & DCIS & 0.8 & 0.4 \\
programmes & Invasive & 4.4 & 1.5 \\
\hline & & &
\end{tabular}

Table 2. Estimated detection rates of non-progressive (i.e. overdiagnosed) and progressive DCIS, and invasive disease, by screening round (prevalence/incidence), pooled estimates from all five programmes in Yen et al. [2].

\begin{tabular}{|c|c|c|c|c|c|}
\hline \multirow[b]{2}{*}{ Screen } & \multicolumn{5}{|c|}{ Detection rate/1000 of } \\
\hline & $\mathrm{DCIS}_{0}$ & $\mathrm{DCIS}_{1}$ & $\begin{array}{l}\text { Invasive } \\
\text { cancer (I) }\end{array}$ & $\begin{array}{l}\mathrm{DCIS}_{0} / \\
\mathrm{DCIS}_{1}\end{array}$ & $\begin{array}{l}\mathrm{DCIS}_{0} / \\
\left(\mathrm{DCIS}_{1}+\mathrm{I}\right)\end{array}$ \\
\hline Prevalence & 0.3 & 0.5 & 5.2 & $37 \%$ & $5 \%$ \\
\hline Incidence & 0.02 & 0.5 & 2.8 & $4 \%$ & $0.6 \%$ \\
\hline
\end{tabular}

$\mathrm{DCIS}_{0}$, non-progressive DCIS; $\mathrm{DCIS}_{1}$, progressive DCIS.
These results suggest firstly that the excess in incidence observed in screened cohorts is followed by a later deficit in invasive tumours in the same cohorts, and secondly that earlier diagnosis of DCIS explains some of the later deficit in invasive disease incidence. This is consistent with results in the Swedish Two-County Study, in which an excess of DCIS cases was observed in the group invited to screening, which was almost exactly balanced by a subsequent deficit of invasive cases in the same population [13].

\section{Discussion}

The modelling approaches described above provide some reassurance that detection of DCIS by screening is valuable and is forestalling the diagnosis of subsequent invasive disease. They suggest that there is some uncertainty about the exact size of the problem of overdiagnosis of DCIS. It would be helpful to see the results of Yen et al. [2] confirmed or refuted by estimation within large screening programmes from individual rather than aggregate data.

There is other published research on overdiagnosis, but not specifically addressing the DCIS question $[14,15]$. Also, these papers tend to concentrate only on observed rates of disease, without formal correction for lead-time effects.

Evidence from the randomized trials is not conclusive, but does not suggest a substantial problem of overdiagnosis of DCIS. Interpretation is complicated by the varying designs of the trials [16].

How do we reconcile the fact that studies of screening programmes suggest modest overdiagnosis only, with the results of the autopsy studies and the follow-up of cases of untreated DCIS? For the first problem, it should be noted that since the autopsy studies found $9-15 \%$ occult DCIS but any screening for the disease finds around $0.1 \%[3,5]$ it is likely that screen-detectable DCIS in living women is either a different clinical entity from, or a small and specialized subset of, autopsy-detectable DCIS in dead women. For the second, the untreated DCIS cases were untreated because they were misdiagnosed as benign [4], not a representative group of DCIS as a whole. Evans and colleagues [8] have shown that screen-detectable DCIS has considerably

Table 3. Excess of breast cancer cases in the Florence programme, with and without adjustment for cases anticipated due to lead time.

\begin{tabular}{lllllll}
\hline Tumours & Observed cases & $\begin{array}{l}\text { Expected from } \\
\text { pre-screening }\end{array}$ & Excess (\%) & $\begin{array}{l}\text { Anticipated } \\
\text { cases }\end{array}$ & $\begin{array}{l}\text { Observed- } \\
\text { anticipated cases }\end{array} \quad$ Excess (\%) \\
\hline Invasive only & 2626 & 2357 & 11 & 215 & 2411 & 2 \\
All tumours & 2780 & 2394 & 16 & 258 & 2522 & 5 \\
\hline
\end{tabular}


greater aggressive potential in terms of grade and necrosis.

In conclusion, the modelling and clinical research on DCIS progression suggests that detection of DCIS in mammographical screening is conferring benefits in terms of invasive disease avoided. While there is a need for further research, the studies suggest that overdiagnosis of DCIS is a minor phenomenon.

\section{References}

1. Peeters $\mathrm{PH}$, Verbeek AL, Straatman $\mathrm{H}$, et al. Evaluation of overdiagnosis of breast cancer in screening with mammography: results of the Nijmegen programme. Int $J$ Epidemiol 1989; 18: 295-299.

2. Yen MF, Tabar L, Vitak B, Smith RA, Chen HH, Duffy SW. Quantifying the potential problem of overdiagnosis of ductal carcinoma in situ in breast cancer screening. Eur $J$ Cancer 2003; 39: 1746-1754.

3. Ernster VL, Barclay J. Increases in ductal carcinoma in situ (DCIS) of the breast in relation to mammography: a dilemma. J Natl Cancer Inst Monogr 1997; 22: 151-156.

4. Page DL, Dupont WD, Rogers LW, Jensen RA, Schuyler PA. Continued local recurrence of carcinoma 15-25 years after a diagnosis of low-grade ductal carcinoma in-situ of the breast treated only by biopsy. Cancer 1995; 76: 1197-1200.

5. Nielsen M, Thomsen JL, Primdahl S, Dyreborg U, Andersen JA. Breast cancer and atypia among young and middle-aged women - a study of 110 medico-legal autopsies. Br J Cancer 1987; 56: 814-819.

6. Bijker N, Peterse JL, Duchateau L, et al. Risk factors for recurrence and metastasis after breast-conserving therapy for ductal carcinoma-in-situ: analysis of European Organization for Research and Treatment of Cancer Trial 10853. J Clin Oncol 2001; 19: 2263-2271.

7. Skinner KA, Silverstein MJ. The management of ductal carcinoma in situ of the breast. Endocr Relat Cancer 2001; 8: 33-45.

8. Evans AJ, Pinder SE, Ellis IO, Wilson AR. Screen detected ductal carcinoma in situ (DCIS): overdiagnosis or an obligate precursor of invasive disease? J Med Screen 2001; 8: 149-151.

9. Feig SA. Ductal carcinoma in situ. Implications for screening mammography. Radiol Clin North Am 2000; 38: 653-668.

10. Morrow M. The certainties and the uncertainties of ductal carcinoma in situ. J Natl Cancer Inst 2004; 96: 424-425.

11. Paci E, Warwick J, Falini P, Duffy SW. Overdiagnosis in screening: is the increase in breast cancer incidence rates a cause for concern? J Med Screen 2004; 11: 23-27.

12. McCann J, Treasure P, Duffy S. Modelling the impact of detecting and treating ductal carcinoma in situ in a breast screening programme. J Med Screen 2004; 11: 117-125.

13. Duffy SW, Tabar L, Vitak B, Day NE, Smith RA, Chen HHT, Yen MFA. The relative contributions of screen-detected in situ and invasive breast carcinomas in reducing mortality from the disease. Eur J Cancer 2003; 39: 1755-1760.

14. Zahl P-H, Strand BH, Maehlen J. Incidence of breast cancer in Norway and Sweden during introduction of nationwide screening: prospective cohort study. BMJ 2004; 328: 921-924.

15. Anttila A, Koskela J, Hakama M. Programme sensitivity and effectiveness of mammography service screening in Helsinki, Finland. J Med Screen 2002; 9: 153-158.

16. Moss S. Overdiagnosis and overtreatment in randomized controlled trials of breast cancer screening. Breast Cancer Res, in press. 